
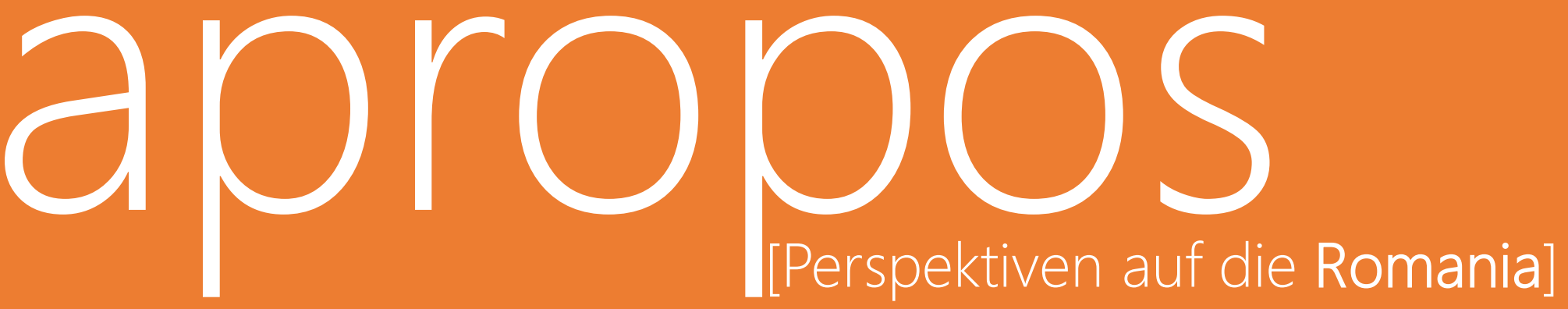

Sprache/Literatur/Kultur/Geschichte/Ideen/Politik/Gesellschaft

\title{
Admirable clarté oder clarté obscure?
}

Der Streit um das génie de la langue française

Martin Gärtner

apropos [Perspektiven auf die Romania] hosted by Hamburg University Press 2021, 6

pp. 113-131

ISSN: 2627-3446

Online

https://journals.sub.uni-hamburg.de/apropos/article/view/1684

Zitierweise

Gärtner, Martin. 2021. „Admirable clarté oder clarté obscure? Der Streit um das génie de la langue française.", apropos [Perspektiven auf die Romania] 6/2021, 113-131. doi: 10.15460/apropos.6.1684

Except where otherwise noted, this article is licensed under a Creative Commons Attribution 4.0 International license (CC BY 4.0)

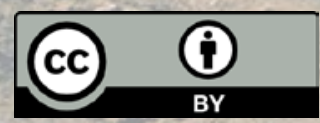

\section{@creative
commons}
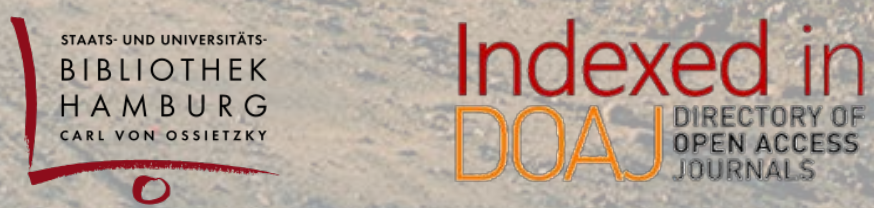


\title{
Martin Gärtner
}

\section{Admirable clarté oder clarté obscure? \\ Der Streit um das génie de la langue française}

\author{
Martin Gärtner \\ ist wissenschaftlicher Mitarbeiter für \\ französische Sprachwissenschaft am \\ Romanischen Seminar der Europa- \\ Universität Flensburg. \\ martin.gaertner@uni-flensburg.de
}

Keywords

Geschichte der französischen Sprache - Sprachapologie - Sprachphilosophie - Sprachdiskussion Nationalsprache

\section{Präliminarien}

Dass die clarté des Französischen ein Topos ${ }^{1}$ bzw. ein Allgemeinplatz in der französischen Sprachdiskussion ist, ist weitgehend bekannt und wird wahrscheinlich jedem Französisch-Studierenden im Laufe seiner Ausbildung vermittelt. Der berühmte Satz von Antoine de Rivarol "Ce qui n'est pas clair, n'est pas français" (Rivarol 1991 [1784], 39) gehört wohl zu den Sätzen, die jeder, der Französisch lernt bzw. studiert, hört und sich merken soll. Unter clarté würde man nun in erster Linie Klarheit verstehen, und tatsächlich ist dies die häufigste Übersetzung, die man für den Begriff clarté im Wörterbuch findet ${ }^{2}$. Doch was bedeutet Klarheit in Bezug auf eine Sprache eigentlich? Bedeutet es, dass die Sprache einer festgefügten Wortfolge im Satz folgt, so wie Rivarol es in seinem Discours sur l'universalité de la langue française im Jahr 1784 erkannt haben wollte? Bedeutet es, dass es wie in den verschiedenen, künstlich geschaffenen Plansprachen des 19. Jahrhunderts, welche als Welthilfssprachen die globale Kommunikation und damit nicht weniger als den Weltfrieden ermöglichen sollten, keinerlei Unregelmäßigkeiten gibt? Kann man Klarheit messen? Sind manche Sprachen klarer als andere? Welche Bedeutung

\footnotetext{
${ }^{1}$ Unter Topos versteht man in der Literaturwissenschaft einen Allgemeinplatz, einen lieu commun, der in der antiken Rhetorik als gedankliches Thema galt, das beliebig weiterentwickelt und abgewandelt werden konnte. Die Topoi entwickelten sich später zu Klischees und erweiterten damit ihren Verwendungsbereich. Dieser Ansicht steht die Idee entgegen, dass Topoi keine frei verwendbaren Klischees sind, welche nach Belieben verwendet werden können, sondern dass sie ihre jeweilige Wirkung erst im Textzusammenhang entfalten. Dementsprechend können Topoi in ihrer Funktion, je nach Absicht des Autors, variabel eingesetzt werden (cf. LWR 2003, 335).

2 Das Lexikon sprachwissenschaftlicher Grundbegriffe des 17. und 18. Jahrhunderts gibt neben dem Wort Klarheit auch die Begriffe Deutlichkeit und Reinigkeit an (cf. Haßler/Neis 2009, 903).
} 
hat das Konzept der clarté heute in der französischen Sprachdiskussion, die seit jeher mit einer deutlich größeren Intensität geführt wird als in anderen europäischen Staaten? Dies sind die Fragen, denen sich dieser Beitrag nähern möchte, ohne den Anspruch zu erheben, alle jemals zur clarté verfassten Texte in den Blick nehmen zu können. Es kann in diesem Zusammenhang nur darum gehen, möglichst repräsentative Texte und Äußerungen zu zitieren, die die Diskussion um die clarté illustrieren. Im Zentrum stehen dabei sowohl historische als auch zeitgenössische Texte und Publikationen, die sich mit dem Französischen befassen und verschiedene Aspekte seiner Klarheit in den Blick nehmen.

\section{Clarté und génie de la langue française}

Wer sich mit dem Begriff der clarté auseinandersetzt - welcher, wie es Mario Wandruszka ausdrückt, zu jenen Begriffen gehört, die als Schlüsselbegriffe das Wesen einer bestimmten Zeit und auch eines ganzen Volkes, jenes der Franzosen, zu beschreiben in der Lage sind (cf. Wandruszka 1959, 135) - wird sehr schnell auf ein weiteres Konzept stoßen, das im Umfeld dieses Begriffs immer wieder auftaucht und ein weiterer Schlüsselbegriff in der französischen Sprachdiskussion ist. Es handelt sich dabei um das génie de la lange française. Hierbei steht man vor dem Problem, dass auch dieser Begriff, ähnlich wie jener der clarté, kontrovers diskutiert wird und eine lange historische Debattengeschichte hinter sich herzieht. Das génie des Französischen zu erklären und zu fassen, ist das Ziel verschiedener Sprachgelehrter, Literaten und Linguisten (z. B. Condillac, Bouhours, Voltaire, Dauzat) gewesen, und auch in der Gegenwart ist das génie de la langue Gegenstand entsprechender Diskussionen. Dass die Definitionen, die zum génie de la langue gegeben werden, voneinander abweichen und dementsprechend verschiedene Aspekte in den Vordergrund stellen, die das Französische und sein Wesen charakterisieren sollen, kann anhand einer selektiven Auswahl an bekannten Äußerungen zum génie de la langue illustriert werden.

Beginnen wir bei Joaquim du Bellay und seiner Deffence, et Illustration de la langue françoyse aus dem Jahr 1549. Diese Schrift, die zu einer Zeit entstand, in der sich das Französische noch wie alle anderen europäischen Volkssprachen der Konkurrenz des Lateinischen als universell gültigem Kommunikationsmittel erwehren musste, beschreibt ausführlich die Höherwertigkeit des Französischen gegenüber dem Griechischen und dem Lateinischen, also gegenüber den beiden klassischen Sprachen der Literatur, Kultur und Politik jener Epoche, wobei insbesondere das Lateinische in Westeuropa eine nicht zu überschätzende Rolle in diesen Bereichen der nicht-alltäglichen Kommunikation einnahm. Du Bellay beschreibt das génie einer jeden Sprache als ein Je ne scay quoi, als etwas Undefinierbares, als eine Art unergründbaren Reiz, den eine jede Sprache ausübt. Da es äußerst individuell ist, können andere Sprachen - z. B. im Rahmen einer Übersetzung - diese Individualität der Einzelsprache nicht wiedergeben. Dementsprechend ist jede Sprache einzigartig und etwas für sich individuell Unnachahmliches (cf. Du Bellay 1991 [1967], 211).

Mit Du Bellay scheint also in Frankreich die Idee in die Welt gekommen zu sein, dass jede Sprache einzigartig ist und dass diese Individualität, also das génie einer jeden 
Sprache, wenn überhaupt, nur schwer zu fassen ist. In den folgenden Jahrhunderten und bis in die Gegenwart hinein beschäftigten (und beschäftigen) sich die Autoren und Sprachgelehrten folgender Generationen mit dem Phänomen des génie de la langue française ${ }^{3}$. Genannt sei hier als Vertreter des 17. Jahrhunderts der Jesuitenpater Dominique Bouhours, der in seinen Entretiens d'Ariste et d'Eugène aus dem Jahr 1671 das Französische in direkten Vergleich zu seinen romanischen Schwestersprachen Italienisch und Spanisch stellt. Abgesehen davon, dass es sich hier um eine eindeutig apologetisch gefärbte Beschreibung des Französischen zuungunsten der beiden anderen Sprachen handelt, stellt Bouhours hier einen klaren Zusammenhang zwischen dem Prestige des Französischen und seiner besonderen Klarheit bzw. Eindeutigkeit her, die in diesem Beitrag interessiert:

Cette naïveté, qui est le propre caractere de nostre langue, est accompagnée d'une certaine clarté, que les autres langues n'ont point. II n'y a rien de plus opposé au langage d'aujourd'huy, que les phrases embarassées; les façons de parler ambiguës; toutes les paroles qui ont un double sens; ces longues parentheses qui rompent la liason des choses; le mauvais arrangement des mots, lorsqu'on ne garde pas bien l'ordre naturel dont nous parlions tout à l'heure, \& qu'on met quelques termes entre ceux qui se suivent naturellement. (Bouhours 2003 [1671], 120)

Das Französische ist dieser Aussage zufolge eine klare, einfache und deshalb besonders zur Kommunikation geeignete Sprache. Sie ermöglicht es, sich eindeutig zu verständigen und hat darüber hinaus den Vorteil, dass sie durch ihre Gestalt und ihre Formen den Boden für einen guten Sprachegebrauch im Sinne Claude Favre de Vaugelas bereitet ${ }^{4}$. Klarheit oder clarté bzw. netteté ist als rhetorische Qualität einer Sprache bzw. von Sprachverwendung keine französische Erfindung. Schon in der Antike wurde die Klarheit, lat. PERSPICUITAS, als Merkmal einer guten rhetorischen Leistung angesehen. Neben dem Aspekt der Klarheit wurden auch andere Merkmale guter Sprachverwendung im Zusammenhang mit rhetorischer Leistung hervorgehoben und seit der Renaissance als humanistische Kriterien der Rhetorik und der Sprachverwendung wiederentdeckt. In einem weiteren Kontext wurden diese Vorzüge der Sprachbetrachtung in Beziehung zu den (angeblichen)

\footnotetext{
${ }^{3}$ Auch in anderen europäischen Staaten bzw. Regionen befassten sich Gelehrte ausführlich mit ihrer jeweiligen Muttersprache und versuchten, ihr Wesen zu ergründen. Diese Überlegungen wurden nicht selten in der Form von Sprachapologien niedergeschrieben, die dazu dienten, die eigene Sprache zu loben und ihre Merkmale positiv hervorzuheben. Einige dieser Schriften waren darüberhinausgehend dazu vorgesehen, andere Sprachen abzuwerten und anhand verschiedenster Kriterien die Überlegenheit der eigenen Mutter- bzw. Nationalsprache zu beweisen. Eine Auswahl solcher sprachapologetischen Schriften bietet z. B. Peter Burke (cf. Burke 2006, 75). Zu den bei inm genannten Apologien gehört aus dem Bereich des Französischen auch La Deffence, et Illustration de la langue françoyse.

${ }^{4}$ Vaugelas ist als remarqueur, also als eine Art Sprachkritiker, im 17. Jahrhundert äußerst einflussreich gewesen und ist bis heute eng mit dem Begriff des bon usage verbunden, welcher seinerseits in der französischen Sprachdiskussion und Sprachkultur eine beispiellose Erfolgsgeschichte durchlaufen hat. Vaugelas, der den bon usage im Vorwort zu seinen Remarques sur la langue françoise aus dem Jahr 1647 als Sprachform des besten Teils des Hofes und der besten Autoren - „la plus saine partie de la cour, conformément à la plus saine façon d'écrire des Autheurs du temps" (cf. Haßler/Neis 2009, 967) - bezeichnete, sorgte mit seinen Vorstellungen vom guten Sprachgebrauch maßgeblich für die Standardisierung des heutigen Französischen und gilt als Wegbereiter des Französischen als Kultursprache.
} 
Mängeln der Sprachverwendung gesetzt. ${ }^{5}$ Die PERSPICUITAS bzw. später die clarté bzw. ,Klarheit' beschreibt seit der Antike eine Vorgabe zur Sprachverwendung, die für die Rhetorik ein zentrales Merkmal darstellt. Klarheit wird dann hergestellt, wenn die Gedanken logisch organisiert sind, um das Verständnis und, wenn nötig, die Überzeugung des Kommunikationspartners zu gewährleisten. Aus dieser Handlungsanweisung zu einem guten und effektiven Sprachgebrauch wird in der Folge seit der Renaissance ein Element für die Apologie der jeweiligen Nationalsprachen, indem - so wie im Falle des Französischen - die Klarheit als besonders ausgeprägtes Element der jeweiligen Sprachen herausgestellt wird (cf. Haßler/Neis 2009, 903). Die Klarheit einer Sprache kann anhand verschiedener Merkmale festgestellt werden. Dazu gehören beispielsweise Charakteristika wie die Natürlichkeit einer Sprache, eine an die Ausführungen von Descartes orientierte Umsetzung der Satzstellung Subjekt-Verb-Objekt bzw. des ORDO NATURALIS ${ }^{6}$, sowie die Annahme, dass eine klare Sprache in der Regel über möglichst wenige Wörter verfügt, da eine klare und eindeutige Sprache sich auch durch einen eher knappen Wortschatz auszeichnet, der eine optimierte, konzise Kommunikation ermöglicht (cf. Haßler/Neis 2009, 916/917). Diese und andere zur Bewertung rhetorischer Aktivitäten bzw. zur Bewertung von Texten entwickelten Kategorien wurden ab dem 16. Jahrhundert zunehmend auf einzelne Sprachen übertragen, sodass eine Vermischung der Ebenen stattfand. Die Bewertung von Texten ging einher mit der nicht selten apologetischen Bewertung von Einzelsprachen. Somit dienten die ursprünglich rhetorischen Kategorien auch dem Sprachvergleich, da dieser ab dem 18. Jahrhundert im größer werdenden Maße über den Vergleich von Texten vollzogen wurde (cf. Schlieben-Lange 1992, 571-572). Speziell in Frankreich wurde die Klarheit als rhetorische Kategorie immer weiter in den Bereich der Grammatik verschoben und damit von der Rhetorik entfernt, sodass in diesem Zusammenhang von einem "Sieg der Logik über die Rhetorik" gesprochen werden kann (cf. Schlieben-Lange 1992, 579). Es bleibt dementsprechend hinzuzufügen, dass sich die Klarheit sowohl auf die Ebene des Diskurses als auch auf die Ebene von einzelsprachlichen Merkmalen beziehen kann, so wie es im Falle des Französischen

\footnotetext{
${ }^{5}$ Zur Beschreibung der Vorzüge und Nachteile einzelner Sprachen gab es seit der Antike ein Gefüge aus verschiedenen Begriffen, welches insbesondere im 18. Jahrhundert erweitert und verändert wurde (cf. Haßler/Neis 2009, 882). Neben der PERSPICUITAS zählen auch Kriterien wie HARMONIA, ABUNDANTIA und ENERGEIA zU diesen humanistischen Sprachkriterien, welche die Vorzüge von Sprachen beschreiben sollen. Einige wie bspw. der Wohlklang bzw. HARMONIA sind eindeutig subjektiv geprägt, andere hingegen können einander widersprechen, so z. B. im Falle des Französischen die Vorzüge der Klarheit und jene des Reichtums. Die Beschreibung von Einzelsprachen mit den Begriffen der Rhetorik ist ein europäisches Phänomen, welches im Zeitraum vom 16. bis zum 18. Jahrhundert seine Blütezeit erlebte. Positive Eigenschaften, die ursprünglich auf Texte verwendet wurden, kamen in jener Zeit zunehmend bei der Beschreibung der europäischen Volkssprachen zum Einsatz (cf. Schlieben-Lange 1992, 571).

${ }^{6}$ Der ORDO NATURALIS spielte bereits in der Antike mit Blick auf die Qualitäten einer guten Rhetorik eine große Rolle. Die Annahme einer bestimmten Wortstellung, welche aus der Natur heraus entsteht, wurde einer künstlichen Wortfolge entgegengestellt (cf. Haßler/Neis 2009, 1134/1135). Der ORDO NATURALIS bezeichnete ebenfalls eine logische Abfolge der Elemente im Satz, während abweichende Satzkonstruktionen eher zur Erzeugung bestimmter rhetorischer Effekte dienten. Im Zuge der Normierungsbestrebungen des Französischen im 17. Jahrhundert wurde der Begriff des orde naturel von den Grammatikern von Port-Royal als ein wesentliches Merkmal insbesondere des Französischen festgelegt (cf. Haßler/Neis 2009, 1136).
} 
insbesondere auf die Satzstellung zutrifft, die (angeblich) eine besondere Klarheit garantiert.

Im 18. Jahrhundert wurde die Diskussion um das génie des Französischen, auch vor dem Hintergrund der Vorstellungen von sprachlicher Klarheit, weitergeführt. Voltaire äußerte sich in seinem Dictionnaire philosophique nicht nur zum génie der Sprachen im Allgemeinen („On appelle génie d'une langue son aptitude à dire de la manière la plus courte et la plus harmonieuse ce que les autres langages expriment moins heureusement" (Voltaire 1879 [1764], 557) und gab Beispiele für die verschiedenen génies der Einzelsprachen. Er orientierte sich mit Blick auf das Französische ebenfalls an der Argumentationslinie Bouhours, da er in seinen Betrachtungen zum génie der Sprachen zu dem Schluss kam, dass sich das Französische insbesondere durch seine Eleganz und durch seine Klarheit auszeichne:

Le français, par la marche naturelle de ses constructions, et aussi par sa prosodie, est plus propre qu'aucune autre à la conversation. Les étrangers, par cette raison même, entendent plus aisément les livres français que ceux des autres peuples. Ils aiment dans les livres philosophiques français une clarté de style qu'ils trouvent ailleurs assez rarement. (Voltaire 1879 [1764], 558)

Den Höhepunkt der Diskussion um die Klarheit des Französischen und damit um seine besondere, individuelle Wesensart markiert allerdings der Discours sur I'universalité de la langue française von Antoine de Rivarol aus dem Jahr 1784. Die Berliner Akademie der Wissenschaften, zu jener Zeit wie andere europäische Institutionen ein Hort des Französischen (cf. Rey 2007, 91), hatte in diesem Jahr in einer ihrer regelmäßig gestellten Preisfragen nach einer Erläuterung der Gründe für die Universalität des Französischen in Europa gefragt. Aus den eingesendeten Preisschriften wurden zwei ausgewählt, die den ersten Platz zugesprochen bekamen: der heute weitgehend vergessene Beitrag von Johann Christoph Schwab und der Discours sur l'universalité de la langue française von Antoine de Rivarol. Rivarol argumentiert in seinem Beitrag maßgeblich mit dem Argument der clarté des Französischen, um die postulierte Universalität dieser Sprache zu begründen. Die clarté beruht dabei in erster Linie auf der festgefügten Wortfolge SubjektPrädikat-Objekt, auch als ordre naturel bezeichnet und bereits in der Antike als ORDO NATURALIS bekannt. Allein das Französische hat, der Auffassung Rivarols folgend, diese seiner Meinung nach natürliche, ursprüngliche und der menschlichen Kommunikation wirklich angemessene Satzkonstruktion als eindeutiges Charakteristikum in sich verankert, auch im Gegensatz zum Lateinischen, von dem es abstammt. Auf diesem Faktum gründet sich seine Universalität und schlussendlich seine Eignung als Universalsprache, d. h. als Sprache der Diplomatie und Politik, der Kunst sowie als Umgangssprache an zahlreichen europäischen, ursprünglich nicht-frankophonen Höfen jener Zeit:

Le français, par un privilège unique, est seul resté fidèle à l'ordre direct, comme s'il était tout raison; et on a beau, par les mouvements des plus variés et toutes les ressources du style, déguiser cet ordre, il faut toujours qu'il existe : et c'est en vain que les passions nous bouleversent et nous sollicitent de suivre l'ordre des sensations : la syntaxe française est incorruptible. C'est de là que résulte cette admirable clarté, base éternelle de notre langue. Ce qui n'est pas clair, n'est pas français ; ce qui n'est pas clair est encore anglais, italien, grec 
ou latin. Pour apprendre les langues à inversion, il suffit de connaître les mots et leurs régimes ; pour apprendre la langue française, il faut encore retenir l'arrangement des mots. On dirait que c'est d'une géométrie tout élémentaire, de la simple ligne droite, que s'est formée la langue française, et que ce sont les courbes et leurs variétés infinies qui ont présidé aux langues grecque et latine. La nôtre règle et conduit la pensée ; [...]. (Rivarol 1991 [1784], 39)

Die Klarheit des Französischen, die, wie Rivarol hier ausführt, auf der eindeutigen, einer geraden Linie folgenden Syntax beruht, betrifft nicht nur die Sprache selbst, sondern auch ihre Sprecher. Das rationale Denken der Franzosen wird folglich zur Grundlage der Klarheit ihrer Sprache. Die Klarheit wurde spätestens im 18. Jahrhundert Teil der Sprachbeschreibung. Ihr Gegenspieler in diesem Bereich war die Qualität der Energie, welche der clarté entgegengesetzt wurde (cf. SchliebenLange 1992, 578). Da die clarté in der Hierarchie der rhetorischen Eigenschaften, die den Einzelsprachen zugeordnet wurden, am höchsten stand, wurde das Französische zwangsläufig als überlegene Universalsprache betrachtet. Wesentlicher Bestandteil dieser Überlegungen war auch in diesem Zusammenhang die Diskussion über die Wortstellung, also den ordre naturel.

Darüber hinaus wurden auch Diskussionen auf politischer Ebene geführt, in denen die Superiorität des Französischen ebenfalls mithilfe des Arguments der Klarheit politisch begründet werden sollte. Auch Rivarol nimmt in diesem Zusammenhang mit seinem Discours an den europäischen Rivalitäten der großen Mächte teil. Während Bouhours im 17. Jahrhundert die Hauptkonkurrenz des Französischen in erster Linie im Italienischen und Spanischen sah, war es im 18. Jahrhundert das Englische, welches auch aufgrund des sich immer weiter ausbreitenden britischen Kolonialismus zunehmend als Hauptrivale des Französischen betrachtet wurde. Die englische Sprache, die man im vorherigen Jahrhundert v. a. als Sprache des Handels betrachtete und der man als solche auch keine weitere Bedeutung beimaß, wurde zunehmend auch auf kulturellem Gebiet zum Rivalen des Französischen, sodass Rivarol in seinem Discours versuchte, das Englische zu diskreditieren und das Französische hingegen als klare und damit universelle Sprache darzustellen (cf. Zollna 2013, 313). Der ordre naturel wird hier - in Anlehnung an Bouhours, aber auch an die Klimatheorie ${ }^{7}$ und den darin behaupteten Zusammenhang zwischen den klimatischen Bedingungen und dem Gemüt eines Volkes - in direkten Zusammenhang zum Charakter der Franzosen gesetzt. Dieser Charakter wäre nach Ansicht Rivarols in erster Linie ein fröhlicher und lebhafter, und dementsprechend müsste sich das Französische eher durch eine lockere, nicht dem ordre naturel folgende Syntax auszeichnen. Entgegen dieser Annahme habe sich aber im Französischen der ordre naturel gut ausgeprägt, mit dessen Hilfe das Französische seine Sprecher förmlich zur Vernunft zwinge. Die Sprache und ihre Strukturen würden folglich den Nationalcharakter in der Form beeinflussen, dass das eigentlich

\footnotetext{
${ }^{7}$ In seinem Essai sur l'origine des langues äußert z. B. Rousseau mit Bezug auf die Klimatheorie, dass die Sprachen des Nordens wegen der dortigen, sehr harten und schwierigen Lebensbedingungen „dures et bruyantes“ (Rousseau 1993 [1781], 99) seien.
} 
eher sprunghafte Denken durch die klare Ordnung der Syntax gezügelt und in fest vorgegebene Bahnen gebracht werde (cf. Zollna 2013, 315).

Aus dieser Sichtweise heraus erklärt sich ein weiterer Schlüsselsatz des Discours von Rivarol, in dem sich die Eigenschaften des Französischen herauskristallisieren, nämlich dann, wenn er das Französische als „sûre, sociale, raisonnable, ce n'est plus la langue française, c'est la langue humaine" bezeichnet (cf. Weinrich 1961, 528). Demgegenüber stehen die Äußerungen des Enzyklopädisten d'Alembert, der sich völlig anders über das Französische äußerte und es, ganz im Gegensatz zu Rivarol, als unklare Sprache charakterisierte. Demzufolge ist die Klarheit nur dann Bestandteil der französischen Sprache, wenn sich der jeweilige Autor bzw. die Sprecher an die stilistischen Vorschriften halten, die die Klarheit sprachlicher Äußerungen garantieren (cf. Weinrich 1961, 530). An diesen Beispielen wird einmal mehr deutlich, dass in der Diskussion um die Klarheit die beiden Ebenen Text und Sprache vermischt werden.

Auch wenn Rivarol mit seinem Discours in die Annalen der französischen Sprachgeschichte eingegangen ist, ist seine Apologie auf das Französische nicht frei von Widersprüchen. Sie wird eher als Aneinanderreihung von Allgemeinplätzen gesehen, welche sich in theoretischer Hinsicht auf widersprüchliche Konzepte und Vorstellungen beziehen. So wird Rivarol beispielsweise dafür kritisiert, dass er Ansichten des Sensualismus und des Cartesianismus miteinander vermengt. Diese Vermischungen betreffen die Aussagen zum ordre naturel und zu dessen Bedeutung für das Französische. Der ordre naturel ist an sich eine rationalistische Sichtweise auf die Syntax und wird von Rivarol auf das Französische dahingehend bezogen, dass es diese Wortfolge von allen Sprachen am besten erhalten habe. Rivarol führt weiter aus, dass auch andere Sprachen diese Konstruktion gekannt, aber diese im Laufe der Zeit aufgegeben hätten, da diese Konstruktion nicht den Empfindungen, den sensations, entsprächen. Diese Argumentation zeigt, dass Rivarol nicht nachvollziehen konnte, dass einerseits der Sensualismus nicht davon ausging, dass die Wortstellung Subjekt-Verb-Objekt nicht die ursprüngliche Satzstellung gewesen sei, und dass andererseits der Rationalismus auch die Abweichung von dieser Ordnung kannte und diese als einen rhetorischen Kunstgriff betrachtete (cf. Christmann 1978, 29-30). Die Anordnung dieser verschiedenen Ideen, die teils bereits widerlegt waren, legen den Eindruck nah, dass die Schrift Rivarols ihre Wirkung eher durch rhetorische Durchschlagskraft erzielen sollte als durch eine in sich stimmige, widerspruchsfreie Argumentation (cf. Haßler/Neis 2009, 919).

Die Universalität und insbesondere die Klarheit des Französischen haben dennoch durch Rivarols Apologie des Französischen endgültig einen Mythenstatus in der französischen Sprachdiskussion erhalten. Dies lässt sich daran ablesen, dass sie bis heute in der französischen Sprachdiskussion präsent sind. Selbst der Name Rivarol scheint sich mittlerweile zu einem eigenen Schlüsselbegriff in dieser Diskussion entwickelt zu haben. Die bloße Nennung seines Namens reicht aus, um Assoziationen mit der angenommenen Universalität des Französischen und seiner clarté zu wecken, und diese Elemente als fest miteinander verbundene Elemente anzuerkennen. Dass z. B. noch Mitte des 20. Jahrhunderts die clarté als 
wesensprägendes Merkmal der französischen Sprache betrachtet wurde, die clarté das Französische zur einzigen wirklich „menschlichen" Sprache machte und dass auch die Abwertung anderer Sprachen unter Zuhilfenahme des clarté-Konzepts begründet wurde, zeigt das Beispiel des französischen Linguisten Jacques Duron. In seinem 1963 veröffentlichten Werk Langue française, langue humaine, welches bereits im Titel eine eindeutige Anspielung auf Rivarol und seinen Discours enthält, bedient sich Duron weitestgehend der Argumentation Rivarols, um die Klarheit und damit die Superiorität des Französischen zu begründen.

\begin{abstract}
S'il est une langue à l'opposé de l'ordre direct, c'est bien l'allemand avec ses inversions, ses propositions emboîtées, sa syntaxe enveloppante et à longue portée. Le déterminant précédant le déterminé, le complément placé avant le verbe et le complément indirect avant le direct, le verbe lui-même éloigné de son auxiliaire et plus encore de son sujet - tous les verbes se massant d'ailleurs à la fin comme des acteurs réunis au baisser du rideau - la proposition principale disloquée par les relatives et suspendue jusqu'à sa résolution finale comme un thème sans cesse interrompu: tout cela, sans parler d'une plus grande liberté de forger des mots composés, fait d'une phrase un peu complexe un labyrinthe musicalement séduisant (car la langue est belle à prononcer, pleine de rythmes et d'accords), mais peu confortable pour qui aime aller d'un pas sûr ; on n'évite de s'y prendre qu'au prix d'une attention entraînée et de maints retours en arrière. Encore, dans la conversation ou dans le discours parlé, l'intonation est-elle là pour guider l'auditeur; mais le texte écrit contraint le lecteur à un effort soutenu, que la ponctuation ne facilite pas beaucoup ; et si déjà cette complexité se marque dans un simple article de journal ou dans un écrit facile, à quel degré n'atteindra-t-elle pas dans des ouvrages de science ou de philosophie! (Duron 1963, 94)
\end{abstract}

Er stellt in dieser Passage ausgiebig die als Mängel interpretierten Merkmale des Deutschen (und an anderer Stelle mit derselben Intention die des Englischen) heraus, um die besondere „Unklarheit" der deutschen Sprache zu illustrieren und sie anhand entsprechender linguistischer Fachtermini wissenschaftlich zu begründen. Das Ergebnis ist ein als ausgesprochen archaisch zu bewertendes Lob der clarté, in dem Duron die französische Klarheit mithilfe der Autoritätsbeweise von Rivarol, aber auch mit Bezug auf die Linguisten Albert Dauzat und Charles Bally, als besonders ausgeprägt beschreibt. Es wird hier versucht, die clarté als Topos der französischen Sprachdiskussion mit allen möglichen linguistischen Argumenten als unumstößliches Faktum zu definieren und damit zumindest indirekt die Überlegenheit des Französischen zu belegen:

Sans méconnaître d'autres caractères importants - la segmentation de la phrase, par exemple, conforme au génie analytique du français - et sans refuser non plus à voir tout ce qui subsiste de souple et même de capricieux dans la construction française, "restée accueillante pour les exceptions tel les variétés susceptibles de rendre les nuances délicates de la pensée", le grand linguiste genevois tenait lui aussi la séquence progressive, ainsi qu'il l'appelait en l'opposition à la séquence anticipatrice caractéristique de l'allemand, pour la norme essentielle de la phrase française, naturellement ennemie de l'inversion ou du moins ne la souffrant que dans des cas exceptionnels. [...] C'est cela même, c'est cette règle fondamentale du français moderne, que Rivarol appelait l'ordre direct. (Duron 1963, 97)

Diese fast an eine Pathologisierung der deutschen und der englischen Sprachen erinnernde Klassifikation und Erläuterung Durons aus den 1960er Jahren folgen ebenfalls der Strategie, die Rivarol bereits im Jahr 1784 verfolgte, nämlich jener, mithilfe des Arguments der Klarheit des Französischen die eigene Sprache auf- und 
andere Sprachen abzuwerten. Das Urteil über die Ausführungen Jacques Durons fiel dementsprechend einige Jahre später im Jahr 1970 sehr eindeutig aus, als Ludwig Söll kommentierte, dass in den Äußerungen Durons der Geltungsanspruch des Französischen in "Dünkel und Maßlosigkeit endet" und dass die "Peinlichkeiten" Durons sich perfekt in die Äußerungen jener Puristen, welche Angst vor dem Verfall und dem Prestigeverlust des Französischen hätten, einreihten (cf. Söll 1983 [1970], 303).

Trotz solcher Aussagen hält sich der Mythos der besonderen Klarheit der französischen Sprache bis heute. Pierre Swiggers bezeichnet sie als Ideologem ${ }^{8}$, als ein „a priori sous-tendant la perception (et l'auto-perception) d'une donnée socioculturelle" (Swiggers 2010, 443). Diese Aussage soll uns nun zu den Stimmen und Argumenten bringen, welche sowohl in den vergangenen Jahrzehnten als auch in der Gegenwart die Diskussion um die clarté und damit um das génie de la langue française geprägt haben und bis heute beeinflussen.

\section{Die clarté des Französischen - Einbildung oder Wirklichkeit?}

Wenn man zeitgenössische Aussagen zur clarté betrachtet und diese z. B. mit den Aussagen Durons kontrastiert, wird man den Eindruck gewinnen, dass die Gültigkeit der clarté zunehmend infrage gestellt wird. Nichtsdestotrotz finden sie weiterhin ihre Verteidiger, sodass sich ein Panorama der aktuellen französischen Sprachdiskussion eröffnet, dass von zwei Polen bestimmt wird. Einerseits wird die clarté bis heute als besondere Qualität des Französischen betrachtet. Gabriel de Broglie, Mitglied der Académie française, drückte dies in einer Rede in Peking im Jahr 2015 folgendermaßen aus:

Les spécialistes des langues décrivent de manière générale les langues par des caractères généraux. L'espagnol est considéré comme une langue noble, l'italien comme une langue harmonieuse, l'allemand comme une langue précise, l'anglais comme une langue naturelle et pour le français on met généralement en avant la qualité de la clarté. (De Broglie 2015)

Andererseits wird in der clarté in erster Linie ein weiterer Baustein zur Mystifizierung der französischen Sprache gesehen, welche wiederum zu ihrer Verknöcherung führt. Hinzu kommen konkrete soziale Verwerfungen, welche durch sprachpuristische Tendenzen hervorgerufen und befördert werden, indem bestimmte Formen des Sprachgebrauchs tabuisiert und stigmatisiert werden. Die Debatte um die clarté zeigt einmal mehr, welchen hohen Stellenwert die Nationalsprache in Frankreich hat und in welchem Ausmaß und mit wieviel Verve die Diskussionen über ihren Gebrauch, ihren Zustand und ihre Zukunft geführt werden ${ }^{9}$. Für Henri Meschonnic, welcher der clarté mit großer Skepsis begegnet,

\footnotetext{
${ }^{8}$ Der Begriff Ideologem stammt von der französischen Literaturwissenschaftlerin Julia Kristeva. Sie definiert das Ideologem als "die gemeinsame Funktion, die eine bestimmte Struktur (sagen wir, den Roman) mit anderen Strukturen (sagen wir, mit dem Diskurs der Wissenschaft) in einem intertextuellen Raum verknüpft. Man bestimmt das Ideologem eines Textes durch seine Bezüge zu anderen Texten“" (Kristeva 1972, 256).

${ }^{9}$ So merkt Paul Cohen zur Bedeutung der Sprache in Frankreich an: „In few countries has language played a greater role in constituting national identity than in modern France. French is first and foremost a politica idiom, enshrined by the leaders of the Revolution and the Third Republic as the language of the Republic and
} 
ist die Vorstellung vom Französischen als besonders klare Sprache - wie er in seinem Essay De la langue française - Essay sur une clarté obscure beschreibt - eine Ansammlung von falschen Ideen und Allgemeinplätzen, hinter der sich keinerlei Substanz verbirgt:

\begin{abstract}
Car parler de la clarté française, généralement, c'est une manière d'exprimer une affection pour cette langue dont on ne saurait douter. C'est justement pour préserver cet affect que, sans trop me faire d'illusions, j'entreprends de débusquer, après bien d'autres, le fatras d'idées fausses ou définitivement arrêtées (la pire chose qui puisse arriver à une idée), qui se fait passer pour la langue française. (Meschonnic 1997, 19)
\end{abstract}

Die Diskussion über die Klarheit ist zwar oberflächlich durchaus als Beweis für ein besonders ausgeprägtes Interesse an der Sprache zu verstehen, aber die falschen Vorstellungen vom Französischen und von seinen Eigenschaften vermitteln ein Bild der Sprache, welches mit der Realität wenig zu tun hat. Auf ähnliche Weise argumentiert Jürgen Trabant, wenn er in seinem Aufsatz „Die Französische Sprache gegen ihr Genie verteidigen. Über Henri Meschonnic: De la langue française. Essai sur une clarté obscure", auch als Reaktion auf die Gedanken Meschonnics, dazu aufruft, die Sprache vor diesen falschen Auffassungen und Ideen zu schützen und gleichzeitig die Personen und Institutionen kritisch zu betrachten, die diese Ideen verbreiten und sie als Existenzgrundlage des Französischen betrachten. Das génie de la langue charakterisiert Trabant als ein äußerst widerstandsfähiges, zeitweise bösartiges Gespenst, das kontinuierlich in der Sprachdebatte erscheint und die Ansichten zum Französischen nachhaltig geprägt hat. Gelänge es, dieses Gespenst zu vertreiben, wäre der französischen Sprache und auch ihren Sprechern seiner Meinung nach ein guter Dienst erwiesen, weil ihre Sprache nicht mehr in ein enges, altmodisches Korsett gezwängt werden würde (cf. Trabant 1999, 3). Alain Rey, einflussreicher französischer Lexikograph, bezeichnet die clarté diesbezüglich in seinem 2007 erschienenen Essay L'amour du français - contre les puristes et autres censeurs de la langue zwar nicht als Gespenst, aber auch er tritt ihr ausgesprochen kritisch entgegen und spricht angesichts der Vorstellungen von sprachlicher Klarheit von einer „ivresse de raison“ (Rey 2007, 90), was einem Generalangriff auf das Französische gleichkommt, wenn man bedenkt, mit welchem Nachdruck die Stellung des Französischen als Sprache der Aufklärung, der Philosophie und des rationalen Denkens gepriesen wurde.

Trabant betrachtet die clarté zudem als explizit nationalen, wenn nicht nationalistischen Mythos. Das Problem der französischen Nationalmythen, welches er konstatiert, besteht darin, dass sie in der Gegenwart immer mehr an Strahlkraft verlieren und eine zunehmend untergeordnete Rolle im gesellschaftlichen Leben spielen (cf. Trabant 1999, 3). Das génie du français bleibt davon nicht verschont. Auf der anderen Seite wird die Diskussion über den Geist des Französischen weiterhin durch eine Vielzahl von Falschdarstellungen und vorgeblichen Wahrheiten, welche sich bei näherer Betrachtung als unwahr erweisen, in die

the Nation. The French state promotes the use of French at home and throughout the world through an array of government institutions, including the Académie française, the Ministry of Culture and the agencies responsible for France's francophonie policies" (Cohen 2000, 21). 
verkehrte Richtung gelenkt. Trabant sprich in diesem Zusammenhang von einem „Panorama des Unsinns", welches er als „kulturell und politisch bornierte, vergangenheitsorientierte, auf ewige Unbeweglichkeit und, Reinheit' ausgerichtete Ideologie der Sprache, die sich der Vielfalt, der Dynamik, der Veränderung, jeder Modernität, kurz der Kreativität der Sprache versagt" (Trabant 1999, 4) bezeichnet. Damit spiele sie den Puristen in die Hände, die das Französische in einem bestimmten Zustand erhalten wollen und jedwede Veränderung ablehnen und als sprachliche Verfallserscheinung brandmarken.

Zu diesen sprachpuristischen Ansichten gehört z. B. die Annahme, dass die Anglizismen, welche ein ebenfalls stark diskutiertes Thema in der französischen Sprachdebatte sind, die Klarheit des Französischen negativ beeinflussen, womit wiederum feststeht, dass der Reichtum einer Sprache nicht unbedingt förderlich für deren Präzision ist. Vor diesem Hintergrund argumentiert z. B. der französische Philologe Maurice Rat, wenn er, wie bereits andere vor ihm, hervorhebt, dass das Französische bzw. die Franzosen unbedingt Klarheit im sprachlichen Ausdruck benötigen und dass diese Klarheit beispielsweise durch Anglizismen beeinträchtigt wird. Die Klarheit ist, und in diesem Zusammenhang orientiert er sich an Rivarol, sowohl ein Wesenszug der Sprache als auch des gesamten Volkes. Demzufolge muss die Sprache in erster Linie Begriffe zur Verfügung stellen, die eindeutig sind und keine Verwirrungen erlauben („,'esprit français, qui aime à saisir du premier coup les objets, n'attache de prix qu'aux pensées qui se dégagent d'une expression nette, ne permettant pas l'équivoque" (Rat 1965, 602)). Diese Klarheit kann nur erreicht werden, wenn die verwendeten sprachlichen Mittel auch wirklich dazu dienen, die Gedanken der Sprecher wiederzugeben, was in der Endkonsequenz auch bedeutet, im Dienst der clarté keine Anglizismen oder andere Lehnwörter aufzunehmen, um dadurch bestehende französische Begriffe zu ersetzen. Klarheit hat in diesem Sinn auch nichts mit dem Reichtum einer Sprache zu tun. Zu viele Wörter schaden der clarté eher, sodass der französische Wortschatz nicht durch übertrieben viele Homonyme oder Synonyme aufgebläht werden soll. Gerade im Bereich der Geisteswissenschaften und der Literatur sieht Rat die Gefahr, dass durch einen Wildwuchs an Wortneuschöpfungen die Klarheit Schaden nehmen könnte (cf. Rat 1965, 604).

Diese unnötigen Unklarheiten wären seiner Meinung nach vermeidbar, wenn man sich konsequent nach den Regeln, die das génie des Französischen vorgibt - obwohl auch Rat hier mit keinem Wort erwähnt, worin das génie des Französischen denn nun eigentlich bestehen soll - der Sprache bedient: „le juste emploi des mots dans la seule proprieté de leur sens contribue à la clarté de la langue“" (Rat 1965, 604). Auch die Idee einer festgefügten Syntax spielt für Rat eine wichtige Rolle. Denn neben der Gefahr durch eine "inflation verbale“ (Rat 1965, 605), die er in der zeitgenössischen Sprache beobachtet, sieht er die clarté ebenfalls durch eine immer weniger eingehaltene Syntax bedroht. Damit meint er genau jene festgefügte Ordnung des Französischen, die spätestens Rivarol als Garant für die Universalität des Französischen festgestellt und prominent vertreten hat. Das Zusammenspiel dieser beiden Faktoren - „inflation verbale“ (Rat 1965, 605) und 
die Auflösung der Syntax - macht es laut Rat immer dringlicher, die Klarheit des Französischen wirksam zu verteidigen:

Si l'on y veut bien joindre une syntaxe de plus en plus lâche, qui tend à substituer dans la langue écrite les approximations ou les pataques de la langue parlée, on aura bref le tableau d'ensemble de différents dangers qui menacent la clarté française, et contre lesquels il nous la faut défendre sur des fronts bien divers. (Rat 1965, 605)

Rat sieht also bereits in den 1960er Jahren einen Verlust an Klarheit und damit an sprachlicher Qualität des Französischen. Er kann mit Fug und Recht in die Reihe derjenigen Sprachpuristen eingeordnet werden, die sich mit Bezug auf Rivarol für eine rigide und hochreglementierte Sprachpflege einsetzten und die das Französische in erster Linie gegen jede Art von Veränderung und Verfall bewahren möchten. Die Gegenstimmen gegen die Auffassung des Französischen als besonders klare Sprache, wie sie Maurice Rat zum Ausdruck bringt, sind im Laufe der Zeit nicht verstummt und scheinen in der Gegenwart eher zahlreicher zu werden. Auch Nichtlinguisten beginnen sich auf der Ebene der Laienlinguistik mit ihrer Sprache auseinanderzusetzen und den Mythos des Französischen, der clarté und des génie de la langue française kritische zu hinterfragen. Dazu gehört Julien Barret. Der französische Autor und Musiker kritisiert in seinem Buch Tu parles bien la France! aus dem Jahr 2016 den Kult, der um das Französische und sein konstruiertes Genie in Form der clarté betrieben wird. Die clarté ist eher Anzeichen eines übersteigerten linguistischen Nationalstolzes:

Quoi qu'il en soit, cette prétendue clarté du français est un préjugé qui manifeste le chauvinisme le plus éclatant - tout comme la thèse selon laquelle le français serait la plus belle langue du monde [...]. (Barret, 2016, 19)

Im Zusammenhang mit der Bedeutung des Französischen für die französische Nation und angesichts der Fixierung auf die clarté als historisches Erbe des Französischen stellen die beiden Soziolinguistinnen Maria Candea und Laélia Véron in ihrer sprachemanzipatorischen Streitschrift Le français est à nous ! aus dem Jahr 2019 die Frage, ob man das Französische lieben könne, ohne nekrophil zu werden, und beziehen dabei Stellung gegen all jene, die das Französische in einem bestimmten Zustand konservieren und damit auch die clarté verteidigen wollen:

Les puristes ont pignon sur rue ; les médias leur tendent très facilement le micro, au point qu'on n'est plus capable de déceler le caractère extrémiste de leurs affirmations. Ils (ce sont généralement des hommes) prétendent, la main sur le cœur et les yeux embués, aimer et défendre le français, toujours présenté comme une victime à la fois magnifique et torturée ; ils affectionnent les métaphores féminines de la langue. Leur verbiage souvent fleuri habille une idée simple et unique : ils aiment un état ancien, disparu du français et ils vomissent le français écrit et oral pratiqué par la quasi-totalité de leurs contemporains, dans les médias, dans la littérature, sur les réseaux sociaux, dans la rue, partout, partout. (Candea/Véron 2019, 81)

Eine eindeutige Gegenposition dazu nimmt wiederum Alain Borer in seinem gegen den Verfall des Französischen gerichtetem Essay De quel amour blessé - Réflexions sur la langue française ein. Er beklagt im Gegensatz zu Candea und Véron, die sich beide explizit gegen einen übertriebenen Purismus wenden, den Zustand des Französischen und hebt im Rahmen eines Sprachvergleichs, ähnlich wie Duron, die 
Syntax des Deutschen negativ hervor. Die nicht den Regeln der französischen Klarheit entsprechende Satzstellung bezeichnet er als Merkmal einer „despotischen“ Grammatik:

On peut se demander ce que produit un tel fait de langue sur des sujets et des peuples qui passent leur temps à attendre le verbe (ou le passage d'un train de mots jusqu'à la locomotive). La langue assujettit, s'il faut se taire pour écouter. Le langage est la relation de pouvoir par excellence, l'instrument d'influence. Mais il en est qui assujettissent plus que d'autres; dont c'est le projet : ce que Hegel appelle die Herrschaft, la domination. Si je donne le verbe tout de suite, je m'expose ; si je garde longtemps le roi dans mon poing caché derrière mon dos, je m'impose. [...] (Borer 2014, 206)

Zwar werden in Borers Ausführungen auch andere Sprachen mit dem Prädikat "despotisch" bedacht, so z. B. das Koreanische. Dennoch kann man an den Äußerungen Borers beobachten, wie der Zusammenhang zwischen dem Denken der Sprecher und den Strukturen einer Sprache in sprachapologetischen Äußerungen gepflegt und weiterhin tradiert wird:

Où est l'autre et quel est-il, la grammaire en décide. La langue française fait place à celui qui peut le faire taire, l'Allemand s'isole. À la différence d'une langue française de la conversation, qui parle avec, les langues allemande, turque, coréenne développent des grammaires potentiellement despotiques. Leurs locuteurs s'en accomodent, parce que les mentalités se constituent à l'intérieur de ce système du diktat qui en empêche l'autocritique. Plus elle est parlée, plus elle se réalise : le Symbolique précède le Réel qui le forme. (Borer 2014, 207)

Abgesehen von diesen Äußerungen, welche das Deutsche und andere Sprachen als Werkzeuge der Despotie und der Unfreiheit eindeutig in Opposition zum Französischen als Sprache der universellen Werte der Französischen Revolution setzen, spricht auch Borer an anderer Stelle in seinem Essay von einem génie de la langue. Dies bezeichnet er allerdings als projet. Das Projekt des Französischen besteht in seinen Augen zwar in erster Linie in einer Form von gemeinsamer Kommunikation, aber auch hier ist die Klarheit zwangsläufig das zentrale Konzept. Auch Borer beantwortet die Frage, warum das Französische nun eine besonders klare Sprache sein soll, weder abschließend noch überzeugend. Eine recht einfache Antwort auf diese Frage könnte Alain Rey geben, wenn er argumentiert, dass die eigene Muttersprache - und über diese schreibt Borer ausführlich in seinem Essay - natürlicherweise immer die klarste Sprache von allen sei, da man diese am besten beherrsche und jede andere, fremde Sprache zwangsläufig unklarer sei (cf. Rey 2007, 97).

\section{Die clarté aus linguistischer Sicht - Sind manche Sprachen klarer als andere?}

Gibt es jenseits der ideologischen und wie eben gesehen durchaus nationalistisch geprägten Sicht auf die clarté des Französischen auch aus der Perspektive der Sprachwissenschaft Argumente für die besondere Klarheit des Französischen? Bei genauerem Hinsehen und angesichts der Ausführungen - auch vonseiten französischer Linguisten - ist dies nicht wirklich der Fall. Wenden wir uns in diesem Zusammenhang Claude Hagège $z u$, der in seiner Sprachgeschichte des 
Französischen, Le français et les siècles, auch auf das Phänomen Rivarol und die universalité bzw. die clarté des Französischen eingeht.

Wesentlicher Bestandteil der clarté ist, wie bereits erläutert, spätesten seit Rivarol der ordre naturel, also die festgefügte Wortfolge Subjekt-Verb-Objekt. Die Überlegungen, dass genau diese Satzstruktur die klarste und logischste ist, reicht bis in die Antike zurück und wurde im Laufe der Jahrhunderte immer wieder diskutiert. Im Fall des Französischen war man spätestens seit dem 17. Jahrhundert, auch vor dem Hintergrund des Cartesianischen Rationalismus, davon überzeugt, dass logische Überlegungen nur durch eine feste Satzfolge ausgedrückt werden können und folglich der ordre naturel die Basis für das logische Denken darstellt (cf. Hagège 1985, 156). Inversionen, wie sie im Französischen ebenfalls vorkommen, galten in diesem Zusammenhang eher als Ausbrüche der Gefühle, und standen damit der Logik im Weg. Die Tatsache, dass seit dem 17. Jahrhundert das Französische auch aufgrund seiner Wortfolge als vollkommen logische Sprache betrachtet wurde, führte auch zu den Annahmen Rivarols, die dieser in seinem Discours zum Ausdruck brachte.

Harald Weinrich erteilt in seinen Ausführungen zur Klarheit des Französischen, die sich auf die Satzstruktur stützen soll, eine klare Absage. Weder die Logik noch die Sprachwissenschaften hätten einen eindeutigen Beweis gefunden, dass eine bestimmte Satzstellung besonders logisch oder eindeutig ist und dementsprechend eine Überlegenheit derjenigen Sprache, die sie befolgt, begründen kann (cf. Weinrich 1961, 537). Die dominante Position des Satzbautyps Subjekt-Verb-Objekt bringt Weinrich eher mit den historischen Entwicklungen in Verbindung, da die Sprache des Âge classique, der französischen Klassik, die ursprünglich vorhandenen syntaktischen Freiheiten des Altfranzösischen aufgegeben hatte und durch bestimmte Vorlieben im Bereich des Sprachstils die festgefügte Wortfolge SubjektVerb-Objekt, also den ordre naturel, zum stilistischen Ideal bestimmt hatte (cf. Weinrich 1961, 536).

Zusätzlich stellt Claude Hagège in Bezug auf die behauptete Klarheit des Französischen - v. a. dann, wenn man damit eine herausgehobene Stellung gegenüber anderen Sprachen postuliert - klar, dass, wenn das Französische wirklich eine absolut klare Sprache wäre, sie sich gegenüber dem Englischen deutlich besser durchsetzen könnte als ihr das in der Gegenwart gelingt. Dennoch sieht Hagège, gerade vor dem Vergleich mit dem Englischen, durchaus eine besondere Klarheit des Französischen. So argumentiert er anhand englischer Zeitungstexte und insbesondere anhand ihrer Titel, dass das Englische aufgrund von Verknappungen, Verkürzungen und Ellipsen ungenaue Äußerungen produziere (cf. Hagège 1987, 170). Der Vergleich mit den französischen Pendants, so Hagège in seiner Argumentation, zeige eine klarere Ausdrucksweise als im Englischen. Gleichzeitig habe das Französische aufgrund dieser Genauigkeit bzw. Klarheit eine Tendenz, z. T. deutlich längere Texte zu produzieren als das Englische, dessen Texte in der Regel deutlich kürzer und prägnanter seien als jene des Französischen. Hagège stellt hier in Bezug auf die Klarheit die Frage, ob nicht gerade die Kürze und Präzision des Englischen eher der Idee der clarté entspricht als die detaillierte Genauigkeit des Französischen. An anderer Stelle äußert sich Hagège hingegen 
kritisch über die Tendenz des Englischen zur Ellipse (cf. Hagège 2013, 145). Diese Tendenz zeigt sich z. B. daran, dass das Englische in bestimmten syntaktischen Zusammenhängen auf die Präpositionen verzichte, in denen das Französische diese zwingend verwenden müsse. Da dies im Französischen nicht möglich ist, sei das Argument berechtigt, dass es sich beim Französischen um eine klare Sprache handelt.

Klarheit ist laut Hagège, trotz aller Überlegungen, dennoch ein in erster Linie an individuelles sprachliches Handeln gebundenes Phänomen. Klarheit ist im Französischen nicht übermäßig stärker ausgeprägt als in anderen Sprachen. Damit sind wir wieder bei der Unterscheidung der Ebenen von Klarheit angelangt. Zum einen existiert Klarheit als Kriterium der Textproduktion, des Diskurses, zum anderen auf der Ebene der Einzelsprache. Wenn man der Idee der clarté etwas Positives anrechnen kann, so Hagège, dann ist es die Tatsache, dass sich eine éthique de la clarté herausgebildet habe, also ein generelles Bemühen um sprachliche Präzision und die Suche nach den dem jeweiligen Kommunikationsanlass angemessenen sprachlichen Mitteln (cf. Hagège 1987, 170). Trotzdem gibt es, um noch einmal auf die Frage nach der clarté als Ergebnis einer bestimmten Satzkonstruktion zurückzukommen, in keiner Sprache eine Wortfolge, die den Aufgaben der Sprache, in erster Linie jenen der Kommunikation, besonders gerecht werden würde. In diesem Zusammenhang verweist Harald Weinrich auf die Verflechtungen innerhalb eines Satzes, die sich durch die Anordnung der verschiedenen Elemente ergeben, und die das Verständnis des Satzinhaltes unabhängig von ihrer horizontalen Abfolge im Satz herstellen (cf. Weinrich 1961, 538).

Schützenhilfe erhalten Hagège und Weinrich angesichts der Widerlegung der linguistischen Beweise für die clarté von Pierre Swiggers, der sich zuerst 1987 und dann 2010 zum génie de la langue, also zur angeblich besonders ausgeprägten Klarheit des Französischen, geäußert hat. Er greift die Argumente auf, die gegen die clarté ins Feld geführt werden, und die clarté entweder als bloßen Mythos oder als Irrtum dahingehend infrage stellen, dass man die sprachliche Leistung des Einzelnen als generelle Qualität der entsprechenden Einzelsprache bewertet (cf. Swiggers 2010, 444). Swiggers verweist auf insgesamt drei Hauptargumente, die gegen die clarté sprechen und den Mythos der sprachlichen Klarheit des Französischen auch aus linguistischer Sicht infrage stellen. Zu diesen drei Argumenten gehören die ambigüités, die dissymétries und die alternances. Unter diesen Kategorien finden sich z. B. die verschiedenen und als willkürlich empfundenen Regeln zum Gebrauch der verschiedenen Pronomen oder auch das Auseinanderfallen von geschriebener und gesprochener Sprache, um nur zwei der besonders prägnanten Probleme zu nennen (cf. Swiggers 2010, 453). All diese Phänomene tragen dazu bei, dass das Französische, wie Swiggers feststellt, kein Paradies für die Klarheit ist (cf. Swiggers 1987, 19). Die Klarheit des Französischen ist, so unterstreicht es auch Swiggers, ein in erster Linie ideologisches Argument, dass dazu dienen sollte (und soll), das Französische gegen andere Sprachen zu verteidigen. Während sie zuerst dazu beitragen sollte, das Französische als dem Lateinischen ebenbürtig zu beschreiben, wurde sie später zum Argument für die 
Überlegenheit des Französischen gegenüber anderen europäischen Sprachen benutzt. Der Begriff der Klarheit selbst ist aus sprachwissenschaftlicher Sicht, entgegen der Analysen und Ausführungen der Verfechter der clarté, eher schwer zu fassen und teilt somit durchaus das Los anderer linguistischer Termini. Letztendlich handelt es sich bei der clarté um ein Konstrukt des menschlichen Geistes, und nicht um ein belegbares sprachliches Merkmal (cf. Swiggers 1987, 5).

\section{Conclusio}

Auch wenn die Sprachwissenschaft keine Beweise für die Existenz einer besonderen Klarheit des Französischen liefern kann, bleibt die clarté als integraler Bestandteil der französischen Sprachdiskussion und der französischen Sprachgeschichtsschreibung erhalten. Dabei ist es zuerst einmal egal, ob sie als sprachwissenschaftliches Faktum verteidigt oder, wie Jürgen Trabant es tut, als Gespenst bzw. Mythos der Überlegenheit der französischen Sprache zurückgewiesen wird. Allein die Tatsache, dass die Notwendigkeit zur Diskussion der clarté und damit des génie de la langue nach wie vor als berechtigt angesehen wird, rechtfertigt nach den Ausführungen dieses Beitrags die Feststellung, dass die clarté als Bestandteil der französischen Sprachkultur und Sprachdebatte ein Erfolgsmodell mit einer reichen Vergangenheit und einer festen Verankerung im französischen Sprachdenken ist. Harald Weinrich fasst die Entwicklung des clarté-Begriffs wie folgt zusammen:

\footnotetext{
Ist mit der Charakterisierung als Mythos nun alles über die clarté gesagt? Keineswegs, wie mir scheint. Denn wir sehen uns bei der clarté vor einem jener heilsamen Mythen, denen Jules Romains in Donogoo-Tonka Denkmäler setzt. Denn die clarté, die im Sprachdenken des Grammatikers Vaugelas jene kühne Verwandlung erfahren hat, hat sich seitdem ein zweitesmal [sic!] verwandelt. Aus einer rhetorischen virtus war sie ein Mythos geworden; aus dem Mythos aber entstand ein sprachliches Ethos, verpflichtend für jeden, der Französisch spricht oder schreibt. Albert Dauzat, der an die clarté glaubte, ist uns Zeuge für diese zweite Verwandlung, wenn er in seinem Guide du bon usage schreibt: La clarté du français impose des devoirs à ceux qui le parlent, plus encore à ceux qui l'écrivent. Adel verpflichtet. (Weinrich 1961, 541)
}

Das Französische an sich ist keine besonders klare Sprache, auch wenn man ihr zubilligen kann, dass im französischen Sprachbewusstsein die Aufforderung, den individuellen Sprachgebrauch möglichst verständlich zu halten, tief verankert ist. Das Französische kann genauso klar und genauso unverständlich sein wie jede andere Sprache auch. Klarheit und Unklarheit hängen nicht vom Sprachsystem, sondern vielmehr vom einzelnen Individuum und vom jeweiligen konkreten Gebrauch einer Sprache ab. Das Gespenst der clarté, so wie es Jürgen Trabant ausdrückte, wird die französische Sprachdiskussion dennoch weiterhin begleiten und die Diskussionen um die clarté werden wohl auch aufgrund ihrer tiefen Verwurzelung in der französischen Sprachgeschichte und des hohen Bekanntheitsgrades ihres berühmtesten Apologeten Antoine de Rivarol nicht abreißen.

\section{Bibliographie}

BARRET, Julien. 2016. Tu parles bien la France! Paris: L'Harmattan.

BORER, Alain. 2014. De quel amour blessé. Réflexions sur la langue française. 
Paris: Gallimard.

BouHOURS, Dominique. 2003 [1671]. Les entretiens d'Ariste et d'Eugène. Éd. établie et commentée par Bernard Beugnot et Gilles Declercq. Paris: Honoré Champion.

BURKE, Peter. 2006. Wörter machen Leute. Gesellschaft und Sprachen im Europa der frühen Neuzeit. Aus dem Englischen von Matthias Wolf. Berlin: Verlag Klaus Wagenbach.

CANDEA, Maria \& Veron, Laélia. 2019. Le français est à nous! Petit manuel d'émancipation linguistique. Paris: La Découverte.

ChristManN, Hans Helmut. 1978. „Antoine de Rivarol und Johann Christoph Schwab. Zwei Stellungnahmen zur Universalität der französischen Sprache." In Studia Neolatina. Festschrift für Peter M. Schon, ed. Thomas, Johannes, 24-37, Aachen: Verlag I.A. Mayer.

COHEN, Paul. 2000. „Of linguistic Jacobinism and cultural balcanization. Contemporary French Linguistic Politics in Historical Context." French Politics, Culture \& Society 18 (2), 21-48.

De Broglie, Gabriel. 2015. La beauté de la langue française. Paris: Académie française.

$<$ http://www.academie-française.fr/la-beaute-de-la-langue-française>

Du BeLLAY, Joachim. 1991 [1967]. Les Regrets. Précédé de Les Antiquités de Rome et suivi de La Défense et Illustration de la Langue française. Préface de Jacques Borel. Édition établie par S. de Sacy. Paris: Gallimard

DURON, Jacques. 1963. Langue française - langue humaine. Paris: Larousse.

HAGEGE, Claude. 1985. L'homme de paroles. Paris: Odile Jacob.

HAGEGE, Claude. 1987. Le français et les siècles. Paris: Odile Jacob.

HAGEGE, Claude. 2013. Contre la pensée unique. Paris: Odile Jacob.

HAßlER, Gerda \& NeIS, Cordula. 2009. Lexikon sprachtheoretischer Grundbegriffe des 17. Und 18. Jahrhunderts. Berlin: de Gruyter.

KRISTEVA, Julia. 1972. „Probleme der Textstrukturation“. In Strukturalismus in der Literaturwissenschaft, ed. Blumensath, Heinz, 243-262, Köln: Kiepenheuer \& Witsch.

LWR=Hess, RAINER, Gustav Siebenmann \& TILBERT Stegmann. 2003. Literaturwissenschaftliches Wörterbuch für Romanisten. Tübingen/Basel: A. Francke Verlag.

MeschonNIC, Henri. 1997. De la langue française - Essai sur une clarté obscure. Paris: Hachette.

RAT, Maurice. 1965. „Clarté française.“ Revue des deux mondes 316, 602-605.

REY, Alain. 2007. L'Amour du français. Contre les censeurs et autres puristes de la langue. Paris: Éditions Denoël.

Rivarol, Antoine de. 1991 [1784]. Discours sur l'universalité de la langue française. Paris: Obsidiane.

RousSEAU, Jean-Jacques. 1993 [1781]. Essai sur l'origine des langues où il est parlé de la mélodie et de l'imitation musicale. Suivi de Lettre sur la musique française et Examen de deux principes avancés par M. Rameauintroduction, notes, bibliographie et chronologie par Catherine Kintzler. Paris: Flammarion.

SCHLIEBEN-LANGE, Brigitte. 1992. „Reichtum, Energie, Klarheit und Harmonie. Die Bewertung der Sprachen in Begriffen der Rhetorik." In Texte, Sätze, Wörter und Moneme. Festschrift für Klaus Heger zum 65. Geburtstag, ed. Anschütz, Susanne R., 571-586, Heidelberg: Heidelberger Orientverlag.

SöLL, Ludwig. 1983 [1970]. „Aspekte der französischen Gegenwartssprache." In Die französische Sprache von heute, ed. Hausmann, Franz Josef, 287305, Darmstadt: Wissenschaftliche Buchgesellschaft.

SWIGGERS, Pierre. 1987. „A I'ombre de la clarté française." Langue française 75, 5-21.

SWIGGERS, Pierre. 2010. „La clarté du français: examen d'un ,idéologème'.“ 
Zeitschrift für Romanische Philologie 126, 434-459.

TRABANT, Jürgen. 1999. „Die französische Sprache gegen ihr Genie verteidigen. Über Henri Meschonnic: De la langue française. Essai sur une clarté obscure." Zeitschrift für französische Sprache und Literatur 109 (1), 1-24.

VoltAIRE, Michel de. 1879 [1764]. „Langues.“ In Dictionnaire philosophique, Vol. 3., CEuvres complètes de Voltaire, Vol. 17, 552-571. Paris: Garnier.

WANDRUSZKA, Mario. 1959. Der Geist der französischen Sprache. Reinbek: Rowohlt.

WEINRICH, Harald. 1961. „Die clarté der französischen Sprache und die Klarheit der Franzosen." Zeitschrift für Romanische Philologie 77, 528544.

ZolLnA, Isabel. 2013. „Vom Sprachstil zum Nationalcharakter. Dominique Bouhours (1671) im Vergleich zu Henri Estienne (1579) und Antoine Rivarol (1784)." Zeitschrift für Romanische Philologie 129, 289-323. 


\title{
Zusammenfassung
}

Die clarté ist ein zentraler Bestandteil der französischen Sprachdiskussion und gleichzeitig hoch umstritten. Spätestens seit Antoine de Rivarol im Jahre 1784 in seinem Discours sur l'universalité de la langue française die clarté als Grundlage für die Universalität des Französischen ins Feld führte, gilt das Französische als wahrhaft klare Sprache par excellence. Die clarté ist bis heute Bestandteil der französischen Sprachkultur und sprachapologetischer Betrachtungen. Doch es mehren sich die Stimmen, die die clarté nicht mehr als uneingeschränkt gültiges Merkmal des Französischen akzeptieren und den Mythos der clarté und damit auch das génie de la langue française zunehmend infrage stellen. Der Beitrag möchte die Entwicklung des clarté-Begriffs skizzieren und die Argumente beleuchten, die in der Gegenwart für und gegen die clarté ins Feld geführt werden. Ebenso soll aus sprachwissenschaftlicher Sicht der Frage nachgegangen werden, ob Klarheit tatsächlich als eine linguistische Kategorie gelten kann.

\begin{abstract}
Clarity (clarté) is a central part of the French language discussion and, however, a highly controversial concept. Ever since Antoine de Rivarol's Discours sur I'universalité de la langue française in 1784, clarity has been used as the basic argument for the universality of French. Since then, French has been regarded as a truly clear language. To this day, the idea of French as an absolutely clear language is an integral part of French linguistic culture and, as such, a component of apologetic considerations. However, there is a growing number of authors who no longer accept clarity as a valid feature of French. These authors increasingly question the myth of the French clarity and thus also the genius of the French language. This article aims to outline the development of the concept of clarity and examine the arguments that are currently being put forward for and against it. Likewise, from a linguistic perspective, the question will be explored as to whether clarity can actually be considered a linguistic category.
\end{abstract}

